\title{
Feasibility Analysis of the Parametric Response Map as an Early Predictor of Treatment Efficacy in Head and Neck Cancer
}

\author{
A.H. Baer, B.A. Hoff, A. Srinivasan, C.J. Galbán, and S.K. Mukherji
}

\begin{abstract}
BACKGROUND AND PURPOSE: Estimating changes in the volume transfer constant, normalized area under the contrast-enhancement time curve at 60 seconds, and fractional blood plasma volume by using dynamic contrast-enhanced MR imaging may be useful in predicting tumor response to chemoradiation. We hypothesized that the parametric response map, a voxel-by-voxel analysis of quantitative dynamic contrast-enhanced MR imaging maps, predicts survival in patients with head and neck cancer.
\end{abstract}

MATERIALS AND METHODS: Ten patients with locoregionally advanced head and neck squamous cell carcinoma underwent definitive concurrent chemoradiation therapy. For each patient, dynamic contrast-enhanced MR imaging data were collected before and 2 weeks after treatment initiation. Change in perfusion parameters within the primary tumor volume with time was analyzed by parametric response mapping and by whole-tumor mean percentage change. Outcome was defined as overall survival. The perfusion parameter and metric most predictive of outcome were identified. Overall survival was estimated by the log-rank test and Kaplan-Meier survival curve.

RESULTS: The volume transfer constant and normalized area under the contrast-enhancement time curve at 60 seconds were predictive of survival both in parametric response map analysis (volume transfer constant, $P=.002$; normalized area under the contrast-enhancement time curve at 60 seconds, $P=.02$ ) and in the percentage change analysis (volume transfer constant, $P=.04$; normalized area under the contrast-enhancement time curve at 60 seconds, $P=.02$ ). Blood plasma volume predicted survival in neither analysis.

CONCLUSIONS: Parametric response mapping of MR perfusion biomarkers could potentially guide treatment modification in patients with predicted treatment failure. Larger studies are needed to determine whether parametric response map analysis or percentage signal change in these perfusion parameters is the stronger predictor of survival.

ABBREVIATIONS: $\mathrm{DCE}=$ dynamic contrast-enhanced; $\mathrm{HNSCC}=$ squamous cell carcinoma of the head and neck; $K^{\text {trans }}=$ volume transfer constant; NAUC $_{60}=$ normalized area under the contrast-enhancement time curve at 60 seconds; PRM $=$ parametric response map; $V_{\mathrm{P}}=$ blood plasma volume

$\mathrm{t}$ is estimated that $>50,000$ new cases of head and neck cancer were diagnosed in the United States in 2013, accounting for $3 \%$ of all new cancer diagnoses. ${ }^{1}$ Worldwide, the disease burden is

\section{Received June 18, 2014; accepted after revision September 16}

From the Department of Radiology (A.H.B., B.A.H., A.S., C.J.G.), University of Michigan Health System, Ann Arbor, Michigan; and Department of Radiology (S.K.M.), Michigan State University, East Lansing, Michigan.

This work was supported by the William N. Hanafee, MD, Research Seed Grant (2011-2012) and the following National Institutes of Health grants: P50CA093990, P01CA059827, P01CA085878.

Paper previously presented in the William N. Hanafee, MD, Research Seed Grant research progress presentation at: Annual Meeting of the American Society of Head and Neck Radiology, October 3-12, 2012; Miami Beach, Florida; and Annual Meeting of the American Society of Functional Neuroradiology, February 17-19, 2014; Miami Beach, Florida.

Please address correspondence to Aaron H. Baer, MD, Massachusetts General Hospital, Division of Neuroradiology, Gray 2, Room 273A, 55 Fruit St, Boston, MA 02114 e-mail: aaronbaer@gmail.com

- Indicates open access to non-subscribers at www.ajnr.org

http://dx.doi.org/10.3174/ajnr.A4296 even greater, with an estimated $5 \%$ of all cancers being those of the head and neck. ${ }^{2}$ Squamous cell carcinoma of the head and neck (HNSCC) accounts for approximately $90 \%$ of primary malignancies in that anatomic region. ${ }^{3-6}$ The overall incidence and rate of death are in decline, but HNSCC continues to present formidable diagnostic and therapeutic challenges. ${ }^{7,8}$

Most HNSCC is advanced (stage III and IV) at the time of diagnosis. ${ }^{9}$ Treatment of locoregionally advanced HNSCC (stages III and IVA/B) commonly involves surgery followed by radiation therapy, surgery followed by concurrent chemoradiation, definitive concurrent chemoradiation therapy, or sequential therapy involving induction chemotherapy followed by concurrent chemoradiation therapy.

Initial posttreatment imaging is performed to identify residual disease, to evaluate the effect of treatment, and to establish a baseline for future comparison. Imaging is generally performed 2-3 months after completion of chemoradiation because transient changes in MR signal characteristics related to acute inflamma- 
Table 1: Subject demographics and disease status at the time of analysis

\begin{tabular}{|c|c|c|c|c|c|c|}
\hline Subject & Age (yr)/Sex & Primary Location & Stage & Status & Survival (mo) & Outcome \\
\hline 1 & $62 / M$ & Tonsil & $\mathrm{T1} N 2 \mathrm{a}$ & NED & 75.4 & Survivor \\
\hline 2 & $65 / F$ & Soft palate & $\mathrm{T} 2 \mathrm{~N} 3$ & LF & 3.5 & Nonsurvivor \\
\hline 3 & $58 / M$ & Hypopharynx & T4 N2b & $\mathrm{LF} / \mathrm{RF} / \mathrm{DF}$ & 10.3 & Nonsurvivor \\
\hline 4 & $83 / \mathrm{M}$ & Larynx & T4 No & LF & 2.0 & Nonsurvivor \\
\hline 5 & $43 / M$ & Tongue base/tonsil & $\mathrm{T} 4 \mathrm{~N} 2 \mathrm{C}$ & DF & 4.6 & Nonsurvivor \\
\hline 6 & $61 / \mathrm{F}$ & Tonsil & $\mathrm{T} 2 \mathrm{~N} 3$ & RF & 66.1 & Survivor \\
\hline 7 & $55 / M$ & Piriform sinus & $\mathrm{T} 2 \mathrm{~N} 2 \mathrm{~b}$ & Unknown & 33.5 & Survivor \\
\hline 8 & $43 / M$ & Tonsil & $\mathrm{T} 2 \mathrm{~N} 2 \mathrm{~b}$ & DF & 18.9 & Nonsurvivor \\
\hline 9 & $57 / M$ & Nasopharynx & $\mathrm{T} 2 \mathrm{~N} 2 \mathrm{~b}$ & NED & 56.4 & Survivor \\
\hline 10 & $60 / M$ & Tongue base & T3 No & NED & 64.9 & Survivor \\
\hline
\end{tabular}

Note:-NED indicates no evidence of disease; LF, local failure; RF, regional failure; DF, distant failure.

tion are considerable in the early postradiotherapy period. ${ }^{10}$ While such an approach is valuable in monitoring treatment response, the delay between initiation of therapy and assessment of response precludes early treatment modification (eg, radiation therapy intensification for poorly responding tumors). ${ }^{11} \mathrm{~A}$ tool that accurately predicts outcome soon after treatment initiation would allow initiation of alternative treatment regimens, reduce unnecessary treatment-related toxicity, and reduce the unnecessary expense of ineffective treatment regimens.

In 2005, the functional diffusion map was introduced, currently termed the parametric response map (PRM). ${ }^{12,13}$ The PRM is a voxel-based approach to analyzing MR imaging data that allows segmentation of a tumor volume on the basis of regional intratumoral changes in the MR signal. ${ }^{12-14}$ The tool was initially applied to ADC maps, but PRM can be applied to any imaging study. PRM analysis may uncover regional intratumoral signal changes that could be masked in whole-tumor mean signalchange calculations.

Dynamic contrast-enhanced (DCE) MR imaging and dynamicsusceptibility contrast MR imaging are techniques capable of estimating tumor vascular permeability, blood volume, blood flow, and extracellular extravascular space. The aims of this study were to prospectively collect DCE MR imaging data from patients with locoregionally advanced HNSCC before and during treatment and to examine the value of PRM analysis and whole-tumor mean change of perfusion parameters in predicting survival.

\section{MATERIALS AND METHODS \\ Patients}

All adult patients (18 years of age or older) presenting to the University of Michigan Health System for treatment of unresectable, locoregionally advanced, biopsy-proved, primary HNSCC for whom radiation therapy was recommended were eligible for inclusion. ${ }^{15}$ Patients were enrolled during a 2 -year period under the supervision of a faculty member of the department of radiation oncology. Informed consent was obtained from all patients, and the study was approved by the internal review board at our institution. Patients who were pregnant at the time of diagnosis or who had known contraindications to MR imaging (eg, ferromagnetic prostheses) were excluded from the study. Patients with dermal or subcutaneous HNSCC involvement were also excluded due to potential susceptibility effects at air-tissue interfaces. Of the 24 patients initially considered for this study, 4 were excluded because of metallic dental hardware noted on CT that was expected to result in unacceptable susceptibility artifacts, 2 patients were excluded after MR imaging for unacceptable susceptibility artifacts that were not anticipated by CT, 1 patient was excluded because of an incomplete treatment course, and 7 patients were excluded due to incomplete or corrupt datasets at the time of analysis. Datasets of 10 patients were analyzed for this study. Table 1 details patient demographics, disease characteristics, and survivorship.

\section{Treatment}

Each patient underwent definitive treatment with fractionated radiation therapy and concurrent chemotherapy. Radiation therapy involved 70 Gy to the primary gross tumor volume and involved lymph nodes for 7 weeks by 3D conformational or intensity-modulated radiation therapy. Initial chemotherapy regimens for the analyzed patients were as follows: 5 patients received weekly carboplatin (area under the curve of 1 ) and paclitaxel (20 $\mathrm{mg} / \mathrm{m}^{2}$ or $\left.30 \mathrm{mg} / \mathrm{m}^{2}\right), 2$ patients received cisplatin $\left(40 \mathrm{mg} / \mathrm{m}^{2}\right)$ weekly, 1 patient received cisplatin $\left(12 \mathrm{mg} / \mathrm{m}^{2}\right)$ daily for 5 days and 5 -fluorouracil $\left(600-\mathrm{mg} / \mathrm{m}^{2}\right.$ continuous infusion) for 5 days on weeks 1 and 5 of radiation therapy, 1 patient received carboplatin (area under the curve of 4 ) every 3 weeks, and 1 patient received cetuximab with a loading dose of $400 \mathrm{mg} / \mathrm{m}^{2}$ followed by a weekly dose of $250 \mathrm{mg} / \mathrm{m}^{2}$. Follow-up involved clinic visits every 6 weeks for the first 24 months, every 3 months for months 25-36, and every 6 months from month 37 onward.

\section{Perfusion MR Imaging Acquisition}

Using a 3T Philips Achieva magnet (Philips Healthcare, Best, the Netherlands), we obtained MR images at 2 time points for each participant-before treatment $\left(t_{0}\right)$ and 2 weeks after initiation of chemoradiation $\left(t_{1}\right)$. Sequences included DCE T1-weighted, preand postcontrast axial T1-weighted, axial T2-weighted FLAIR, axial T2-weighted with fat saturation, and diffusion-weighted. Each DCE acquisition included 32 dynamic volumes acquired by a 3D gradient-echo pulse sequence following the intravenous administration of $0.1 \mathrm{~mL} / \mathrm{kg}$ of gadopentetate dimeglumine. DCE data were acquired in the sagittal plane to include the entire primary tumor volume and suspected metastatic lymph nodes in the same FOV, while minimizing the acquisition time and the FOV itself. We used the following acquisition parameters: $\mathrm{TR}=5.1 \mathrm{~ms}$, $\mathrm{TE}=1.1 \mathrm{~ms}$, flip angle $=20^{\circ}$, voxel size $=2 \times 2 \times 2 \mathrm{~mm}$.

\section{Defining Tumor Volumes}

Primary gross tumor volumes of interest were manually defined on $t_{0}$ DCE MR imaging data by using masking software developed 


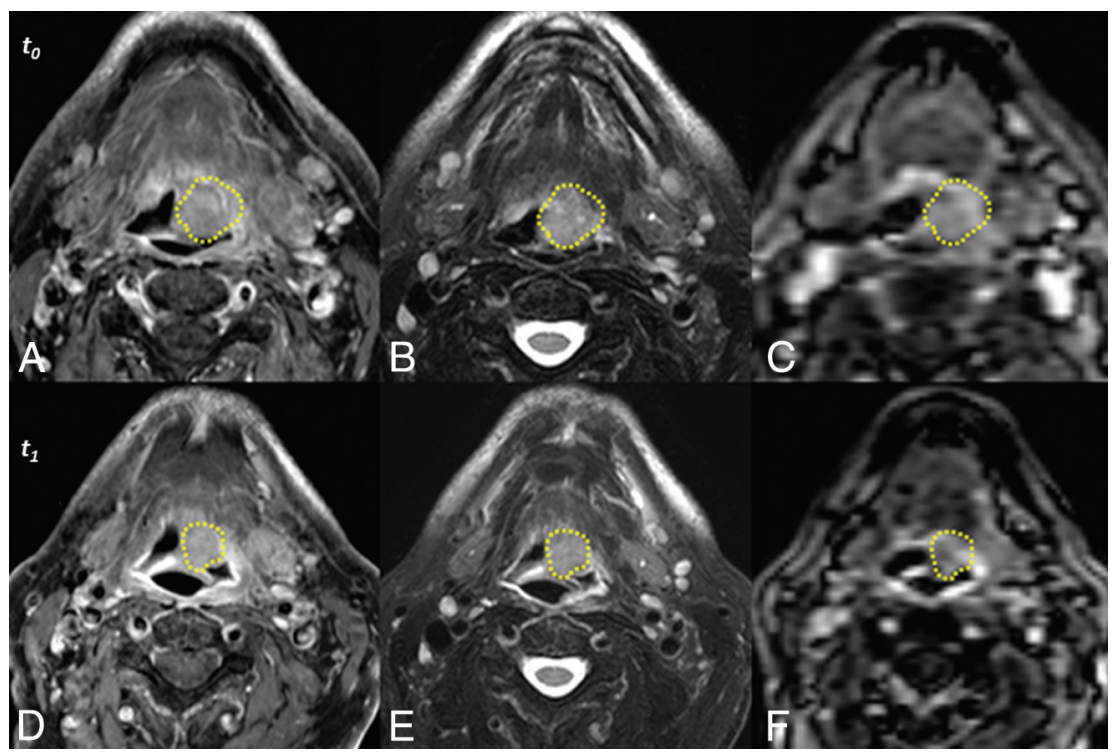

FIG 1. Unregistered sequences of a representative subject acquired before treatment $\left(t_{0}\right.$, top row) and 2 weeks after initiation of therapy ( $t_{1}$, bottom row). Acquisitions include postcontrast T1-weighted with fat-saturation ( $A$ and $D)$, T2-weighted with fat-saturation ( $B$ and $E$ ), and DCE MR imaging ( $C$ and $F$ ). Manually defined primary gross tumor volumes are depicted for each time point (yellow lines). The images above are those of subject 10, a survivor at follow-up.

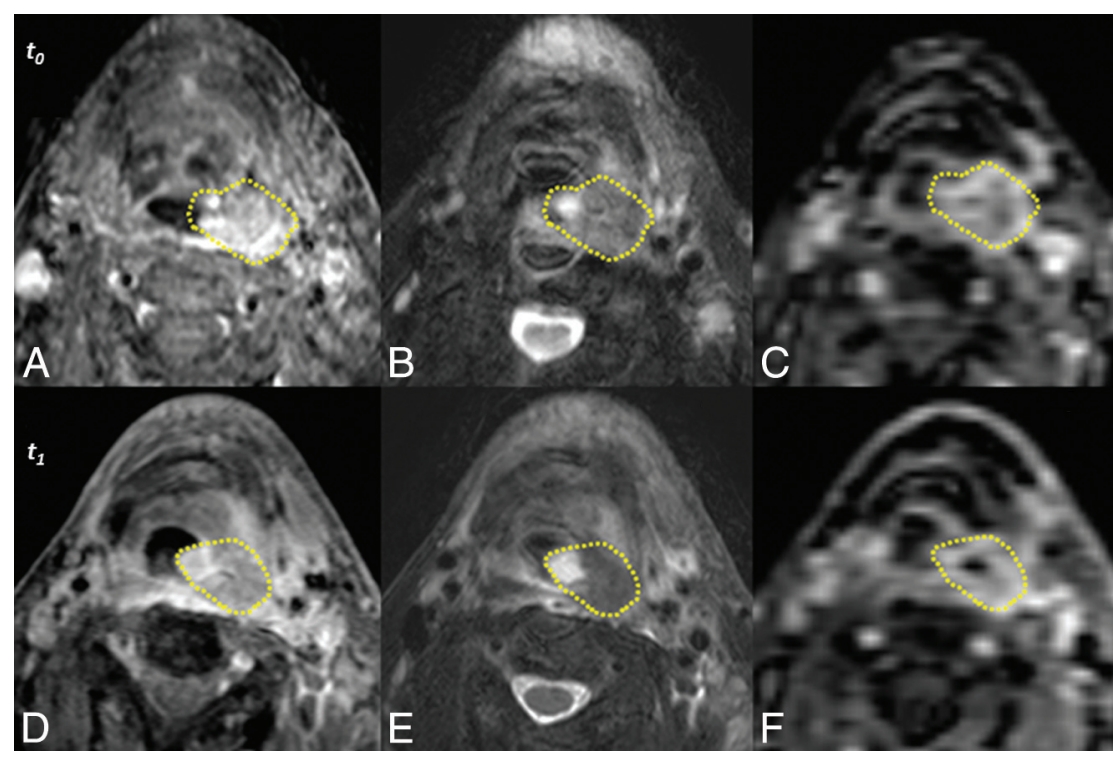

FIG 2. Unregistered sequences of a representative subject acquired before treatment $\left(t_{0}\right.$, top row) and 2 weeks after initiation of therapy ( $t_{1}$, bottom row). Acquisitions include postcontrast T1-weighted with fat-saturation ( $A$ and $D$ ), T2-weighted with fat-saturation ( $B$ and $E$ ), and DCE MR imaging $(C$ and $F)$. Manually defined primary gross tumor volumes are depicted for each time point (yellow lines). The images above are those of subject 3, a nonsurvivor at follow-up. the primary tumor, and samples without clear arterial waveforms were manually excluded from the calculation. The modified Tofts model was used to extract perfusion parameters from the DCE MR imaging data at both $t_{0}$ and $t_{1}$ time points. ${ }^{16}$ For the purposes of this study, parameters of interest include the volume transfer constant $\left(K^{\text {trans }}\right)$, the normalized area under the contrast-enhancement time curve at 60 seconds $\left(\mathrm{NAUC}_{60}\right)$, and blood plasma volume $\left(\mathrm{V}_{\mathrm{p}}\right)$.

\section{Whole-Tumor Change in Perfusion Parameters}

Signal values were extracted from each voxel of the primary gross tumor volume, and averaging these provided whole-tumor mean signal values. The percentage signal change was then calculated by subtracting the whole-tumor mean signal of $t_{0}$ and $t_{1}$ and dividing by that of $t_{0}$. This process was completed for each perfusion parameter $\left(K^{\text {trans }}\right.$, $\mathrm{NAUC}_{60}$, and $\mathrm{V}_{\mathrm{p}}$ ) of each patient. With the parameter $K^{\text {trans }}$ as an example, the resulting whole-tumor mean signal change was reported as a percentage increase $\left(\% \Delta_{\text {Ktrans }}\right)$ or percent decrease $\left(\% \Delta_{\text {Ktrans- }}\right)$.

\section{Parametric Response Mapping}

Coregistration of DCE MR imaging data from $t_{0}$ and $t_{1}$ time points was executed by using the Mutual Information for Automatic Multimodality Image Fusion software package developed at our institution. In brief, $t_{0}$ and $t_{1}$ DCE MR imaging data for each patient were spatially aligned by using thin-plate splines as the deformable registration interpolant. Registration was initiated by manually defined control points in the $t_{1}$ dataset that corresponded to regions within the primary tumor volume as defined on the $t_{0} \mathrm{da}$ - at our institution. Lymph nodes and major vascular structures were excluded from the volumes of interest. Postcontrast T1weighted fat-saturated and T2-weighted fat-saturated sequences were referenced to assist in defining the primary tumor volumes (Figs 1 and 2). For volume-of-interest definition and for comparison with conventional MR images, the DCE MR imaging volumes were reformatted into the axial plane.

\section{Extraction of Perfusion Parameters}

To determine the arterial input function, we sampled the DCE MR imaging signal from the internal carotid artery ipsilateral to taset. The automatic algorithm then iteratively optimized the solution by using mutual information as the objective function.

Following coregistration, the temporally resolved DCE MR imaging data occupy the same $3 \mathrm{D}$ space, with each voxel consisting of a pair of parameter values at the 2 discrete time points. Individual voxels were classified on the basis of the change in the voxel parameter values. Those voxels with a significant increase in parameter $\mathrm{X}$, which may be $K^{\text {trans }}, \mathrm{NAUC}_{60}$, or $\mathrm{V}_{\mathrm{p}}$, were colorcoded red, voxels with a significant decrease were blue, and those without a significant change in X were green. Global PRM mea- 


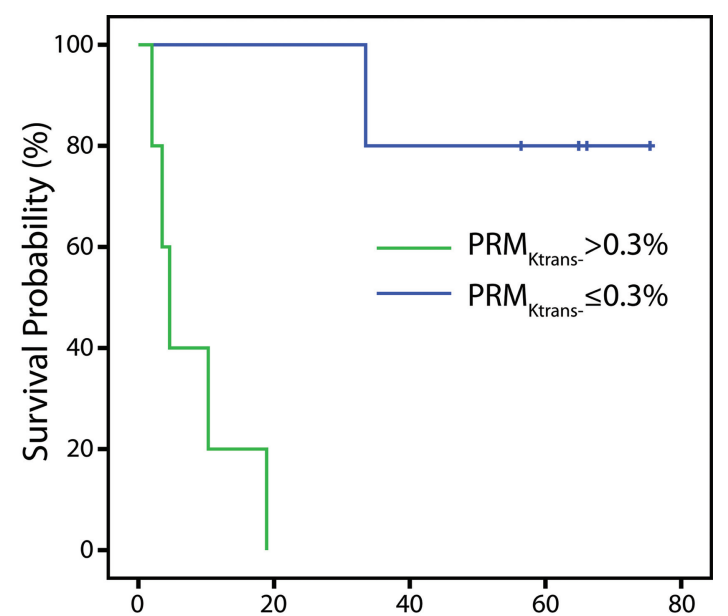

A Time post-treatment initiation (months)

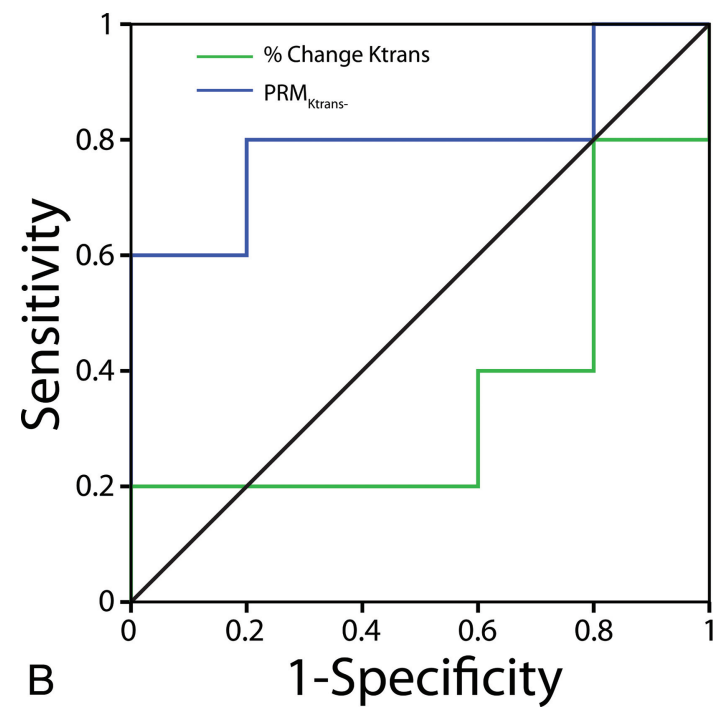

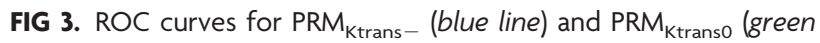
line) at 2 weeks after treatment initiation (A). Kaplan-Meier survival plots for overall survival as a function of stratification by PRM Ktrans- $_{-}$ (the perfusion parameter most predictive of survival) at 2 weeks after treatment initiation $(B)$. The blue line represents $\mathrm{PRM}_{\mathrm{Ktrans}-} \leq$ the cutoff. The green line represents PRM $_{\text {Ktrans }-}>$ the cutoff.

sures were determined by summing all parameter voxels within a classification and normalizing by the total tumor volume. These measures are presented in $\mathrm{PRM}_{\mathrm{X}+}$ for increasing, $\mathrm{PRM}_{\mathrm{X}}-$ for decreasing, and $\mathrm{PRM}_{\mathrm{Xo}}$ for unchanged.

\section{Statistical Analysis}

Outcome was defined as overall survival, the duration of which was calculated from the day of pretreatment MR imaging to the time of data analysis. An optimal "responder cutoff" was identified by using a receiver operating characteristic analysis for each parameter $\left(K^{\text {trans }}\right.$, $\mathrm{NAUC}_{60}$, and $\mathrm{V}_{\mathrm{p}}$ ), analyzed by using either PRM or the percentage change in the mean, and the parameter value most predictive of outcome was identified. Overall survival was estimated by the log-rank test and Kaplan-Meier survival curve (Fig 3).

\section{RESULTS}

At the time of analysis, 5 patients were survivors (50\%) and 5 were nonsurvivors (50\%). For survivors, median survival duration was
Table 2: Statistical significance of analysis type and perfusion parameter in predicting survival

\begin{tabular}{|c|c|c|c|c|c|c|}
\hline & \multicolumn{2}{|c|}{$K^{\text {trans }}$} & \multicolumn{2}{|c|}{$v_{p}$} & \multicolumn{2}{|c|}{ NAUC $_{60}$} \\
\hline & & $\%$ & & $\%$ & & $\%$ \\
\hline & PRM- & Change & PRM- & Change & PRM- & Change \\
\hline Overall survival & $.002^{a}$ & $.041^{a}$ & .308 & .068 & $.022^{\mathrm{a}}$ & $.024^{\mathrm{a}}$ \\
\hline
\end{tabular}

64.9 months (range, 33.5-75.4 months); for nonsurvivors, median survival duration was 4.6 months (range, 2.0-18.9 months).

Table 2 details the statistical significance of each analysis type for each metric in predicting overall survival. For the parameter $K^{\text {trans }}$, both PRM analysis $\left(\mathrm{PRM}_{\mathrm{K} \text { trans-}}, P=.002\right)$ and percentage change analysis $\left(\% \Delta_{\text {Ktrans }-}, P=.04\right)$ were predictive of survival. Similar results were observed for $\mathrm{NAUC}_{60}\left(\mathrm{PRM}_{\mathrm{NAUC60}-}, P=\right.$ $.02 ; \% \Delta_{\text {NAUC60- }}, P=.02$ ). Irrespective of analytical technique, $\mathrm{V}_{\mathrm{p}}$ was not found to be predictive of survival $\left(\mathrm{PRM}_{\mathrm{Vp}^{-}}, P=.31\right.$; $\left.\% \Delta_{\mathrm{Vp}^{-}}, P=.07\right)$. The sample size was insufficient to determine whether PRM analysis or percentage change in perfusion parameters provided a stronger predictor of survival. Figure 4 depicts sample sections from $\mathrm{PRM}_{\mathrm{Ktrans}}$ in a representative survivor and nonsurvivor.

\section{DISCUSSION}

Recently, PRM analysis of perfusion data has been examined as a surrogate biomarker for early cancer treatment response. In 2009, Galbán et $\mathrm{al}^{17}$ compared PRM analysis with whole-tumor mean percentage analysis of CBV and CBF in predicting treatment response in high-grade gliomas. Results indicated that PRM analysis of CBV and CBF within the tumor were highly predictive of survival after 1 week of treatment, whereas percentage change analysis was not predictive. Furthermore, PRM of CBV was more predictive of overall survival than baseline CBV. In 2010, Tsien et $\mathrm{al}^{18}$ examined PRM analysis of perfusion data to distinguish between disease progression and pseudoprogression in high-grade gliomas undergoing concurrent chemoradiation therapy. The study found that at week 3 of therapy, patients with progressive disease had a significant decrease in PRM of CBV compared with patients with pseudoprogression. In 2012, Wang et al ${ }^{19}$ evaluated an analysis method known as "fuzzy clustering" to identify HNSCC tumor subvolumes in DCE MR imaging data related to treatment outcomes. Results suggested that tumor subvolumes with low blood volume that persisted from pretreatment to intratreatment (week 2 of concurrent chemoradiation therapy) time points were greater in patients with local treatment failure than in those with local control.

In our study, both $\mathrm{PRM}_{\mathrm{Ktrans}-}$ and $\% \Delta_{\mathrm{Ktrans}-}$ were predictive of survival when measured early after treatment initiation. Patients with a large percentage of the primary gross tumor volume that decreased in $K^{\text {trans }}$ (ie, a measure of endovascular permeability) were more likely to have significantly reduced survival. Similarly, findings suggest that patients with a large percentage of the primary gross tumor volume that decreased in $\mathrm{NAUC}_{60}$ (a general measure of perfusion) were more likely to have significantly reduced survival. These findings are consistent with those previously described.

The clinical implication of our findings is that PRM is feasible and may identify, early into definitive treatment, those patients whose primary tumors will have an unfavorable response to stan- 

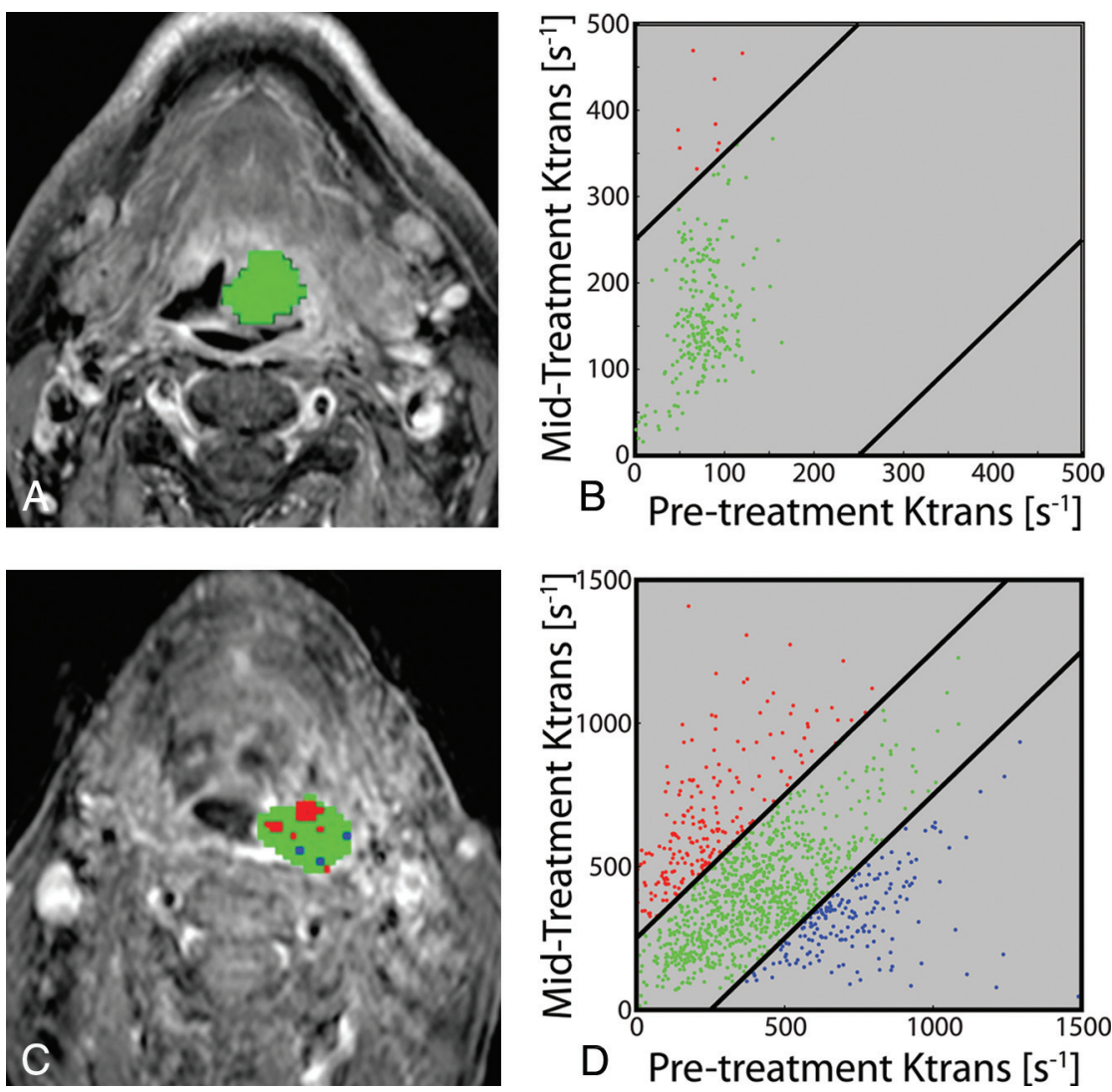

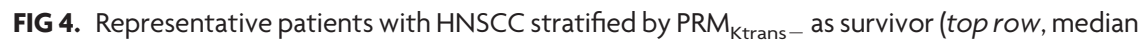
survival time, 64.9 months) or nonsurvivor (bottom row, median survival time, 10.3 months) at the

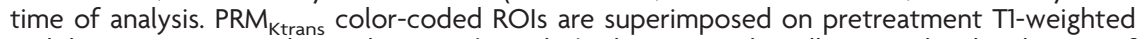
gadolinium contrast-enhanced images $(A$ and $C$ ). The scatterplots illustrate the distribution of changes in $K^{\text {trans }}$ throughout the entire volumes of interest $(B$ and $D)$. The $95 \%$ confidence intervals within the scatterplots are represented by the black lines. Voxels with significantly increasing, decreasing, or unchanged $K^{\text {trans }}$ are coded as red, blue, and green dots, respectively.

dard chemoradiation regimens. Perhaps even more useful, PRM provides spatial information regarding the location of unresponsive tumor subvolumes, which may aid in selecting targets for radiation therapy intensification. The converse may also be true-tumor subvolumes with a predicted favorable response to standard radiation doses may have a similarly favorable response to a reduced dose.

\section{Study Limitations}

The processing requirements of the PRM metric are not trivial, and the modeling of DCE MR imaging data has yet to be standardized. A critical question when considering implementation of such an analysis is whether the PRM metric provides a stronger predictor of survival than percentage whole-tumor mean signal change, a simpler and less time-consuming analysis. The sample size of this feasibility study is insufficient for the multivariate analysis needed to determine which analysis type produces a stronger predictor of outcome.

A $128 \times 128$ in-plane matrix size was used for the DCE MR imaging acquisition in this study because using large matrices in the 32-phase sequence is inherently time-consuming. The resulting low spatial resolution may have compromised accurate manual definition of primary gross tumor volumes of interest, particularly for smaller lesions. To avoid inclusion of voxels that were subject to volume averaging in the analysis, we used conservative margins in defining the volumes of interest. This use likely resulted in undersampling signal in the periphery of the primary tumor volumes.

Data collection for this study was part of a larger investigation involving conventional and diffusion MR images. The perfusion technique had motion in some individuals, was incomplete in others due to scanner failure, and was corrupted in others likely during transfer to network storage. These issues were addressed and are not anticipated in planned future trials.

Finally, measuring the arterial input function remains a substantial source of error in the estimation of perfusion parameters with DCE-MR imaging. The optimal method of reproducibly estimating the arterial input function (eg, cohort average versus measured patient-specific) remains the subject of debate. $^{20}$

\section{CONCLUSIONS}

The results of this retrospective analysis of prospectively collected data suggest that PRM analysis of perfusion biomarkers could potentially guide early modification of chemoradiation treatment regimens. Specifically, subvolumes of tumors with predicted treatment failure may benefit from radiation therapy intensification early in the treatment course. A prospective investigation is currently taking place at our institution to examine targeted radiation therapy intensification with the application of our technique. Larger studies are also needed to validate these findings and to determine whether PRM analysis or whole-tumor percentage signal change analysis provides a stronger predictor of survival.

\section{ACKNOWLEDGMENTS}

We gratefully acknowledge Dr Thomas Chenevert for his invaluable assistance in the preparation of this article.

Disclosures: Aaron H. Baer-RELATED: Grant and Provision of Writing Assistance, Medicines, Equipment or Administrative Support: The American Society of Head and Neck Radiology supported the study described in the article through the William N. Hanafee research seed grant $(\$ 15,000)$.* The funds were used for the purchase of equipment used in the study and as salary support for 2 of the coauthors, Benjamin A. Hoff and CraigJ. Galbán. Ashok Srinivasan—Royalties: Amirsys (for book chapters). Benjamin A. Hoff-RELATED: Grant: National Institutes of Health. * Craig J. GalbánRELATED: Grant: National Institutes of Health (P50CA093990, P01CA059827, P01CA085878)*; UNRELATED: Patents (planned, pending or issued): University of Michigan (coinvestor of this technology); Royalties: University of Michigan, Imbio, Comments: As a coinventor of this technology, I may receive royalties from the licensing of this technology to Imbio from the University of Michigan. Suresh K. Mukherji-RELATED: Grant: American Society of Head and Neck Radiology (Hanafee Award).* *Money paid to the institution. 


\section{REFERENCES}

1. Siegel R, Naishadham D, Jemal A. Cancer statistics, 2013. CA Cancer J Clin 2013;63:11-30

2. Ferlay J, Shin HR, Bray F, et al. Estimates of worldwide burden of cancer in 2008: GLOBOCAN 2008. Int J Cancer 2010;127:2893-917

3. Crozier E, Sumer BD. Head and neck cancer. Med Clin North Am 2010;94:1031-46

4. Cohan DM, Popat S, Kaplan SE, et al. Oropharyngeal cancer: current understanding and management. Curr Opin Otolaryngol Head Neck Surg 2009;17:88-94

5. Lin DT, Cohen SM, Coppit GL, et al. Squamous cell carcinoma of the oropharynx and hypopharynx. Otolaryngol Clin North Am 2005;38: 59-74, viii

6. Osborne RF, Brown JJ. Carcinoma of the oral pharynx: an analysis of subsite treatment heterogeneity. Surg Oncol Clin N Am 2004;13:71-80

7. American Cancer Society. Cancer Facts \& Figures 2010. http://www. cancer.org/research/cancerfactsstatistics/cancerfactsfigures2010/ index. Accessed February 3, 2014

8. Jemal A, Siegel R, Xu J, et al. Cancer statistics, 2010. CA Cancer J Clin 2010;60:277-300

9. Monnerat C, Faivre S, Temam S, et al. End points for new agents in induction chemotherapy for locally advanced head and neck cancers. Ann Oncol 2002;13:995-1006

10. Hermans R. Post-treatment imaging of head and neck cancer. Cancer Imaging 2004;4(Spec No A):S6-15

11. Cao $Y$. The promise of dynamic contrast-enhanced imaging in radiation therapy. Semin Radiat Oncol 2011;21:147-56

12. Hamstra DA, Chenevert TL, Moffat BA, et al. Evaluation of the functional diffusion map as an early biomarker of time-to-progression and overall survival in high-grade glioma. Proc Natl Acad Sci U S A 2005;102:16759-64
13. Moffat BA, Chenevert TL, Lawrence TS, et al. Functional diffusion map: a noninvasive MRI biomarker for early stratification of clinical brain tumor response. Proc Natl Acad Sci U S A 2005;102:5524-29

14. Galbán CJ, Mukherji SK, Chenevert TL, et al. A feasibility study of parametric response map analysis of diffusion-weighted magnetic resonance imaging scans of head and neck cancer patients for providing early detection of therapeutic efficacy. Transl Oncol 2009; 2:184-90

15. Srinivasan A, Chenevert TL, Dwamena BA, et al. Utility of pretreatment mean apparent diffusion coefficient and apparent diffusion coefficient histograms in prediction of outcome to chemoradiation in head and neck squamous cell carcinoma. J Comput Assist Tomogr 2012;36:131-37

16. Tofts PS, Brix G, Buckley DL, et al. Estimating kinetic parameters from dynamic contrast-enhanced $\mathrm{T}(1)$-weighted MRI of a diffusable tracer: standardized quantities and symbols. J Magn Reson Imaging 1999;10:223-32

17. Galbán CJ, Chenevert TL, Meyer CR, et al. The parametric response map is an imaging biomarker for early cancer treatment outcome. Nat Med 2009;15:572-76

18. Tsien C, Galbán CJ, Chenevert TL, et al. Parametric response map as an imaging biomarker to distinguish progression from pseudoprogression in high-grade glioma. J Clin Oncol 2010;28:2293-99

19. Wang P, Popovtzer A, Eisbruch A, et al. An approach to identify, from DCE MRI, significant subvolumes of tumors related to outcomes in advanced head-and-neck cancer. Med Phys 2012;39: 5277-85

20. Lavini C, Verhoeff JJ. Reproducibility of the gadolinium concentration measurements and of the fitting parameters of the vascular input function in the superior sagittal sinus in a patient population. Magn Reson Imaging 2010;28:1420-30 